\title{
Le patient peut-il exiger des prestations du médecin?
}

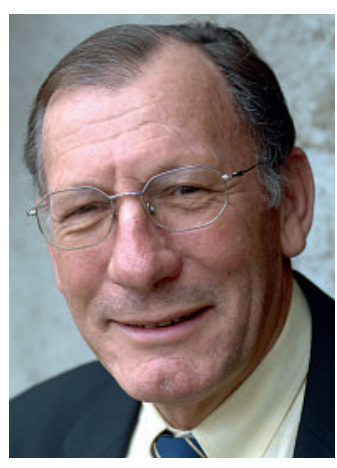

Jean Martin
Les décennies récentes ont été placées sous le signe des «droits des patients», avec une évolution marquée dans les relations soignant-soigné. Comme médecin cantonal, j'ai participé aux développements y relatifs. Un point majeur qui a été souligné, et ancré dans les textes législatifs, a trait au droit plein et entier du patient d'accepter ou de refuser les soins qui lui sont proposés. Condition indispensable pour l'exercice de cette autonomie: l'information préalable, suffisante et compréhensible du malade par le médecin ou autre soignant, qui doit être systématique et spontanée pas fournie seulement quand le patient la demande.

C'est un vrai progrès. Mais tout progrès peut donner lieu à interprétation erronée ou à revendication excessive. Alors qu'il faut dire que le malade a le droit de poser à son médecin n'importe quelle question et n'importe quand, il peut arriver que certains fassent un usage inconsidéré, sans fin, de cette prérogative. Dans la foulée, il arrive que le patient, raisonnant peutêtre par analogie avec son droit à l'information, réclame/exige du thérapeute telle prestation, une prescription médicamenteuse ou un autre geste médical. Ces demandes peuvent concerner des interventions chirurgicales (on pense à la césarienne pratiquée sur demande de la femme enceinte..., l'espace ne permet pas ici de développer ce point de l'évolution de la médecine), ou des mesures «héroïques» dans des circonstances où elles sont considérées comme du registre de la «medical futility». A relever aussi le domaine des certificats médicaux de complaisance - il y en a.

$\mathrm{Qu}^{\prime}$ en est-il donc? Le droit d'exiger un traitement serait-il un jumeau de celui de les refuser? La réponse est non. Le malade est en droit d'attendre que le médecin lui propose toutes les mesures diagnostiques, thérapeutiques ou préventives que demande et justifie le «state of the art» dans son cas. Mais il ne saurait, sur la base de son seul désir ou de sa seule lecture de sites web, exiger que le praticien réalise des actions médicales si ce dernier les juge inutiles.

Une des raisons qui requièrent cette règle est le besoin de pratiquer une médecine économique mais ce n'est pas la plus importante: le premier élément est déontologique, en rapport avec le «primum nil nocere» hippocratique. On rappelle souvent qu'il n'y a pas de geste médical sans risque, aussi modeste ce risque soit-il. Or, c'est le professionnel qui dispose des compétences scientifiques et d'expérience pratique
Well... Cela est bel et bon me dira-t-on mais, dans une ambiance sociétale de plus en plus consumériste, où le praticien peut craindre de perdre son patient s'il n'obtempère pas à ses demandes, cette déontologie tient-elle encore? Elle devrait. Dans les soins médicaux, le «client» ne peut pas être le roi comme il est censé l'être ailleurs, même si à vrai dire certaines évolutions commercialisantes ressemblent à un business comme un autre. On peut évoquer diverses prestations esthétiques pratiquées par des médecins (en fait, la question ici serait de savoir si elles entrent dans le cadre de la médecine à proprement parler ou plutôt du confort et du «lifestyle» - étant admis que des médecins ont le droit cas échéant d'exercer des activités extérieures au domaine médical). Dans tous les cas, on souhaite que la médecine garde une dimension éthique non-mercantile.

Il convient encore de faire une distinction importante: s'il l'estime non justifiée, le médecin doit refuser de fournir toute prestation par complaisance ou gain de paix. Par contre, il ne peut refuser une prestation à laquelle, pour une raison ou l'autre, le patient a droit. Exemple: le médecin doit refuser d'établir un certificat médical sur un point qu'il n'a pas observé personnellement ou qu'il ne connaît pas suffisamment pour se déterminer; par contre il n'a pas le droit, par exemple, de refuser un certificat au patient qu'il a soigné et qui en a besoin pour le présenter à une assurance ou à une autre instance.

Noter que la logique ci-dessus s'applique au domaine des directives anticipées, inscrites à fin 2008 dans le Code civil suisse: ces directives sont contraignantes pour le médecin et l'équipe soignante, qui sont tenus de les observer. Par contre, elles ne sauraient exiger d'eux des actes médicalement inacceptables ou contraires à la loi.

Reste à rappeler la question de l'objection de conscience: le professionnel de santé peut refuser de pratiquer un acte que sa conscience réprouve, mais à certaines conditions: d'abord, qu'il ne s'agisse pas d'une urgence mettant en danger la santé ou la vie de la personne qui consulte; ensuite, il a le devoir d'orienter celle-ci vers un confrère susceptible de l'aider [1].

Jean Martin, membre de la rédaction et de la Commission nationale d'éthique \footnotetext{
et c'est une conduite critiquable de sa part que d'accepter de fournir des prestations non-indiquées voire contre-indiquées.

Martin J. Objection de conscience: déontologie et service à la population. Bull Méd Suisses. 2008;89(24):1104
} 